\title{
DERMATITE FLAGELADA INDUZIDA POR BLEOMICINA: RELATO DE CASO
}

Walfrido Bispo Junior', Rosane Soares Oliveira², Silvana Maria Barros de Oliveira ${ }^{3}$, Monik Kelly Santos Lima ${ }^{4}$

${ }^{1}$ Farmacêutico. Doutor em Biotecnologia em Saúde. Hospital Universitário Professor Alberto Antunes. Maceió-AL-Brasil. ${ }^{2}$ Farmacêutica. Especialista em Saúde do Adulto e do Idoso. Hospital Universitário Professor Alberto Antunes. Maceió-AL-Brasil. ${ }^{3}$ Enfermeira. Mestranda em Enfermagem. Hospital Universitário Professor Alberto Antunes. Maceió-AL-Brasil. ${ }^{4}$ Enfermeira. Especialista em Oncologia. Hospital Universitário Professor Alberto Antunes. Maceió-AL-Brasil.

RESUMO: A Dermatite Flagelada consiste de uma hiperpigmentação cutânea de aspecto flagelado, tendo como causa específica a bleomicina. A bleomicina é um agente quimioterápico usado no tratamento de diferentes neoplasias. O objetivo deste relato é descrever o caso de uma paciente de 31 anos de idade, em tratamento de Linfoma de Hodgkin com protocolo de Quimioterapia Antineoplásica composto por quatro drogas, dentre elas, a bleomicina, que cursou com o desenvolvimento da Dermatite Flagelada. Trata-se de um relato de caso ocorrido num hospital universitário do nordeste do Brasil em 2014. A terapia utilizada como tratamento foi uso de corticoide, o que proporcionou a remissão das lesões do tipo flagelo e permitiu a continuidade do tratamento sem necessidade de mudança do protocolo quimioterápico. A importância do estudo se deve por levantar discussão sobre os aspectos que envolvem o manejo dessa hipersensibilidade, para garantir a continuidade do tratamento antineoplásico. DESCRITORES: Erupção por droga; Protocolos de quimioterapia combinada antineoplásica; Doença de Hodgkin.

\section{BLEOMYCIN-INDUCED FLAGELLATE DERMATITIS: A CASE REPORT}

\section{DERMATITIS FLAGELADA INDUCIDA POR BLEOMICINA: RELATO DE CASO}

\begin{abstract}
Flagellate Dermatitis consists of cutaneous hyperpigmentation with a flagellate appearance, one specific cause of which is bleomycin. Bleomycin is a chemotherapy agent used in the treatment of different neoplasias. This report's objective is to describe the case of a 31-year-old patient receiving treatment for Hodgkin Lymphoma with a protocol of Antineoplastic Chemotherapy made up of four drugs, including bleomycin, which led to the development of Flagellate Dermatitis. This is a report of a case which took place in a teaching hospital in the Northeast of Brazil in 2014. The therapy used as treatment was use of corticosteroid, which led to the remission of the lesions of the flagellate type and allow the continuation of the treatment without the need for changing the chemotherapy protocol. The study's importance lies in that it raises discussion regarding the aspects involving the management of this hypersensitivity so as to ensure the continuity of the antineoplastic treatment.
\end{abstract}

DESCRIPTORS: Drug eruption; Antineoplastic combined chemotherapy protocols; Hodgkin Lymphoma.
RESUMEN: La dermatitis flagelada consiste en una hiperpigmentación cutánea de aspecto flagelado y su causa específica es la bleomicina. La bleomicina es un agente quimioterápico usado en el tratamiento de diferentes neoplasias. El objetivo de este relato fue describir el caso de una paciente de 31 años de edad, en tratamiento de Linfoma de Hodgkin con protocolo de Quimioterapia Antineoplásica compuesto por cuatro drogas, entre las cuales la bleomicina, ocasionada con desarrollo de Dermatitis Flagelada. El caso ocurrió en un hospital universitario del nordeste del Brasil en 2014. La terapia utilizada como tratamiento fue uso de corticoide, lo que proporcionó la remisión de las lesiones del tipo flagelo y permitió la continuidad del tratamiento sin necesidad de cambio del protocolo quimioterápico. El papel del estudio es abrir discusión acerca de los aspectos que involucran el manejo de esa hipersensibilidad, para garantizar la continuidad del tratamiento antineoplásico.

DESCRIPTORES: Erupción por droga; Protocolos de quimioterapia combinada antineoplásica; Enfermedad de Hodgkin.

\section{Autor Correspondente:}

Walfrido Bispo Junior

Hospital Universitário Professor Alberto Antunes

Av. Lourival MeloMota, $\mathrm{s} / \mathrm{n}^{\circ}$-57072-900-Tabuleirodos Martins-AL-Alagoas

E-mail: walfridobispojr@hotmail.com
Recebido: $11 / 01 / 2015$ Finalizado: 15/04/2015 


\section{INTRODUÇÃO}

A bleomicina é uma mistura de antibióticos glicopeptídeos produzidos pela fermentação do Streptococcus verticillus, que possuem atividade antitumoral contra carcinoma escamoso do colo do útero, tumores testiculares e linfomas, como é o caso do Linfoma de Hodgkin ${ }^{(1)}$. Sua eficácia é comprovada por sua atuação que culmina na morte celular. Isso ocorre pela produção de radicais livres que rompem as cadeias de DNA das células cancerígenas ${ }^{(2)}$.

Com a diminuição do uso da bleomicina nos protocolos de citostáticos a Dermatite Flagelada (DF), uma reação adversa rara, ficou sendo escassamente documentada ${ }^{(3)}$. A literatura descreve a DF como uma forma peculiar de hiperpigmentação de aspecto linear que remete a lembrança de "chicotadas", que pode derivar de uma inflamação e que geralmente acomete o tronco ${ }^{(4)}$, o dorso ${ }^{(5-6)}$, a superfície palmar e plantar $^{(3)}$.

Embora pouco relatada, além das alterações pigmentares, a bleomicina também pode causar a gangrena digital. Porém, a DF ainda é a menos comum dessas lesões alcançando uma incidência entre $8-20 \%$ conforme descrito pela comunidade científica ${ }^{(3)}$. Por estas considerações é possível inferir a necessidade de documentar casos dessa reação adversa típica do uso da bleomicina para aumentar a produção de conhecimento sobre essa questão, favorecendo a troca de experiências sobre o manejo dessa toxicidade. E neste sentido, objetiva-se relatar um caso de DF ocorrido em um hospital universitário do nordeste brasileiro.

\section{RELATO}

M. I. A. S., do sexo feminino, 31 anos, parda, professora, portadora de Linfoma de Hodgkin, em tratamento com doxorrubicina, bleomicina, vinblastina e dacarbazina (ABVD), tratada no primeiro semestre de 2014, apresentou placas eritematosas e pruriginosas em face, tronco e membros, dias após a realização do primeiro ciclo de quimioterapia em um hospital universitário do estado de Alagoas. As lesões caracterizadas por hiperpigmentação iniciaram-se nos membros superiores. Logo de início excluiu-se a suspeita de extravasamento quimioterápico local visto que ambos os membros estavam comprometidos e em apenas um havia sido administrado a quimioterapia via endovenosa.

Foi prescrito Bactrim $\AA$, Hixizine $₫$, Diprisone $\AA$ tópico e encaminhada para avaliação pela dermatologia. Paciente relatava melhora discreta das placas eritematosas com o uso de anti-histamínicos, desaparecendo quase que totalmente as lesões dos membros superiores, porém houve progressão para o tronco, dorso e região cervical com o curso dos ciclos de quimioterapia.

Ao exame dermatológico apresentava placas eritemato-violáceas, algumas descamativas, outras com vesículas e bolhas na superfície, de configuração linear, disseminadas no pavilhão auricular, membros e tronco. Foi prescrito Prednisona e Hixizine, em seguida foi realizada biópsia e imunohistoquimica. O resultado da biópsia mostrou pele com erosão focal, parenquimatosa com escamocrosta e espongiose do epitélio, derme com moderado infiltrado inflamatório misto perivascular composto por numerosos histiócitos, eosinófilos e linfócitos, sem exocitose.

Estes achados correspondem à reação de hipersensibilidade tipo farmacodermia, a qual pode ser causada pela bleomicina. Após este diagnóstico levantou-se a hipótese de DF. A paciente foi liberada para realização dos outros ciclos de quimioterapia mantendo associado o uso de um corticoide (Prednisona).

A imunohistoquimica confirmou a hipótese inicial levantada com o resultado da biópsia. O tratamento com anti-histamínicos e corticoides resultou na remissão parcial da DF enquanto mantida sob o uso da bleomicina e a remissão total ocorreu após quatro semanas do término do tratamento citostático.

\section{DISCUSSÃO}

A DF recebe este nome por assumir uma forma linear que lembra lesões do tipo flagelo provocada por chicotadas. Esta lesão cutânea é induzida pela bleomicina e se inicia cerca de 12 a 24 horas após a exposição a essa droga citostática ${ }^{(2)}$. A relação entre a causa e a dose ou a via de administração ainda está pouco estabelecida. Porém, há quem defenda que uma dose cumulativa de 
bleomicina de 16-286 mg por via endovenosa ou intraperitoneal pode ser desencadeadora da reação de hipersensibilidade que leva a $\mathrm{DF}^{(7)}$. No caso descrito, o início das lesões se deu após o primeiro ciclo de infusão de bleomicina.

O metabolismo da bleomicina é realizado pela enzima hidrolase que apresenta baixos níveis nos tecidos pulmonares e na pele, levando a inferência de ser esta, a razão desses tecidos serem acometidos das reações adversas como é o caso da $\mathrm{DF}^{(8)}$. Outra possível causa dessa toxicidade sobre a pele pode ser pequenos traumas como escoriações ou pressão sobre áreas de proeminências ósseas, ou mesmo o prurido, que podem provocar aumento do fluxo sanguíneo resultando numa maior concentração de bleomicina nessas áreas, processo desencadeador das lesões ${ }^{(9)}$.

Ainda sobre essa questão, embora a bleomicina seja administrada em protocolo (ABVD) de poliquimioterapia para Linfoma de Hodgkin, a DF nãoé relacionada às outras drogas desseprotocolo ${ }^{(9)}$.

O resultado da biópsia e da imunohistoquímica foi compatível com uma reação do tipo inflamatória hiperpigmentosa como a DF em conformidade com o que descreve a literatura nos aspectos de espongiose, degeneração da camada basal, infiltrado inflamatório linfo-histiocitário na derme, melanófagos na derme papilar e vasculite linfocítica sem alteração epidérmica ${ }^{(4)}$.

Poucos são os relatos que informam o manejo da DF, porém o uso de anti-histamínicos e corticoides tópico e oral já foram descritos como capazes de trazer benefícios pela diminuição do prurido ${ }^{(9)}$. Referente ao desaparecimento das lesões há autores que estabelecem um período de cinco semanas após término do tratamento com a bleomicina ${ }^{(3)}$ ou após descontinuidade da exposição à droga ${ }^{(7)}$.

O uso de corticoides e anti-histamínicos foi utilizado no caso descrito, havendo diminuição progressiva das lesões, permitindo a continuidade do tratamento sem a necessidade de mudança de protocolo.

\section{CONCLUSÃO}

A bleomicina já é reconhecida como a droga citostática capaz de desencadear a DF que se configura por lesões disseminadas na pele de aspecto hiperpigmentoso, pós-inflamatório, linear do tipo flagelo provocado por pseudo chicotadas, que geralmente acomete o tronco, o dorso, os membros, as superfícies palmar e plantar e que carece de maiores informações quanto ao manejo que favoreça a continuidade do tratamento, diminuindo os prejuízos sobre a terapia antitumoral.

\section{REFERÊNCIAS}

1. Brunton L, Parker K, Blumenthal D, Buxton, I. Goodman \& Gilman: Manual de Farmacologia e Terapêutica. 11a ed. Porto Alegre: AMGH; 2010. Agentes Antineoplásicos. p. 853-908.

2. Gangas CS, Tuma MC, Osses MS. Eritema flagelado por bleomicina, A propósito de un caso. In Reunião Anual de Dermatologistas Latino-Americanos; 2014, Maio, Santiago de Chile, Chile; 2014.

3. Biswas A, Chaudhari PB, Sharma P, Singh L, Julka PK, Sethuraman G. Bleomycin induced flagellate erythema: revisiting a unique complication. Journal of Cancer Research and Therapeutics. 2013;3(9):500-3.

4. Kondo RN, Pontello-Júnior R, Pontello R. Dermatite flagelada: relato de caso. Revista Brasileira de Medicina. 2012;69(2):31-3.

5. Manoj J, Kaliyadan F, Dharmaratnam AD. Palmar and flagellate hyperpigmentation following low dose intralesional injection of bleomycin for cystic hygroma. Dermatol Online J. 2008;14(8):19.

6. Maciá ES, Guillén PC, Rodriguez LA, Laita UM, Carrato MA. Varón con enfermedad de Hodgkin con exantema vesiculoso de distribución simétrica en manos tras tratamiento quimioterápico. An. Med. Interna. 2006;23(5):252-3.

7. Peçanha, C.; Constanza, G.; Dacier, B.; Sodré, C. T. Flagellate dermatitls by bleomycin [apresentação ao Curso de Especialização em Dermatologia da Universidade Federal do Rio de Janeiro; 2007; Rio de Janeiro, Brasil].

8. Diao DY, Goodall J. Bleomycin-induced flagellate dermatitis. Canadian Medical Association Journal. 2012;184(11):1280.

9. Mota GD, Penna AMD, Soares RC, Baiocchi OCG. Bleomycin-induced flagellate dermatites. Rev. Bras. Hematol. Hemoter. 2014;36(4):297-9. 